\title{
Neonatal Silver-Russell syndrome assumed to result from maternal uniparental heterodisomy of chromosome 7
}

\author{
Yoongu Kang ${ }^{\oplus}$, Jinsup Kim ${ }^{\oplus}$, Hyun Ju Lee ${ }^{\oplus}$, and Hyun Kyung Park ${ }^{\oplus}$ \\ Department of Pediatrics, Hanyang University Medical Center, Hanyang University College of Medicine, Seoul, Korea
}

\begin{abstract}
Silver-Russell syndrome (SRS) is a rare genetic disorder characterized by intrauterine growth restriction, poor postnatal growth, relative macrocephaly, a triangular face, body asymmetry, and feeding difficulties. It is primarily diagnosed according to a clinical scoring system; however, the clinical diagnosis is confirmed with molecular testing, and the disease is stratified into the specific molecular subtypes. SRS is a genetically heterogeneous condition. The major molecular changes are hypomethylation of imprinting control region 1 in 11 p15.5 and maternal uniparental disomy of chromosome 7 (UPD(7)mat). Therefore, first-line molecular testing should include methylation-specific approaches for these regions. Here, we report an extremely low birth weight (ELBW) infant with intrauterine growth retardation, postnatal growth retardation, and dysmorphic facial appearance-characteristics consistent with the clinical diagnostic criteria of SRS. Methylation-specific molecular genetic analysis revealed UPD(7)mat, while the loss of heterozygosity was not detected on chromosomal microarray analysis. We present a case of SRS with suspected uniparental heterodisomy of chromosome 7 in an ELBW infant.
\end{abstract}

Key words: Silver-Russell syndrome, Uniparental disomy, Genomic imprinting, Infant, Extremely low birth weight infant.

\section{Introduction}

Silver-Russel syndrome (SRS) is a rare but well-recognized imprinting disorder mainly characterized by idiopathic intrauterine growth retardation (IUGR) and postnatal growth failure. Children with SRS can be distinguished from those with idiopathic IUGR and postnatal growth failure but without SRS by the presence of other characteristic features, including relative macrocephaly, a prominent forehead, body asymmetry, and feeding difficulties. Furthermore, the less consistent clinical features include a triangular face, micrognathia, low muscle mass, and fifth-finger clinodactyly. The incidence of this disorder ranges from 1 in 3,000 to 1 in 100,000 individuals [1]. A clinical diagnosis of SRS is primarily established on the basis of a combination of characteristic features as reflected in the Netchine-Harbison clinical scoring system (NH-CSS) [2]. However, the diagnosis of SRS can be difficult owing to the variations in the manifestation of the condition among the affected individuals and several features of SRS are nonspecific.

Molecular testing can confirm the diagnosis of SRS in approximately $60 \%$ of the cases, and the molecular subtype can be defined, which may aid in selection of the appropriate manage-

Received: 30 May 2020, Revised: 24 July 2020, Accepted: 24 July 2020, Published: 31 December 2020

${ }^{*}$ Corresponding author: Jinsup Kim, M.D. (iD https://orcid.org/0000-0002-9641-5192

Department of Pediatrics, Hanyang University Medical Center, Hanyang University College of Medicine, 222 Wangsimni-ro, Seongdong-gu, Seoul 04763, Korea.

Tel: +82-2-2290-8385, Fax: +82-2-2290-8385, E-mail: pinees@gmail.com

Conflict of interest: The authors declare that they do not have any conflicts of interest.

(ㄷ) This is an open-access article distributed under the terms of the Creative Commons Attribution Non-Commercial License (http://creativecommons.org/licenses/by-nc/4.0/) which permits unrestricted non-commercial use, distribution, and reproduction in any medium, provided the original work is properly cited.

(c) Copyright 2020 by the Korean Society of Medical Genetics and Genomics 
ment strategies. SRS is a genetically heterogeneous disorder; the loss of methylation (LOM) at imprinting control region (ICR) 1 in 11 p 15.5 is detected in up to $40 \%$ of the cases and maternal uniparental disomy of chromosome 7 (UPD[7]mat) accounts for 7-10\% of the cases. Other rare molecular genetic or cytogenetic abnormalities have also been identified [2]. Methylation-specific approaches for chromosomes 7 and 11 are recommended as the first-line tests for the genetic diagnosis of SRS. In particular, methylation-specific polymerase chain reaction (MS-PCR) for chromosome 7 and methylation-specific multiplex ligation probe-dependent analysis (MS-MLPA) for chromosome 11 have been suggested $[3,4]$. For infants with IUGR, an early genetic diagnosis can improve the medical outcomes through the implementation of specialized management strategies, such as administration of growth hormone therapy and early interventions for neurocognitive problems [1]. Here, we report a case of SRS with suspected uniparental heterodisomy of chromosome 7 in an extremely low birth weight (ELBW) infant with IUGR.

\section{Case}

A preterm female infant was born at a gestational age of 27 weeks and 4 days via an emergency cesarean section performed because of severe maternal pregnancy-induced hypertension. She was a naturally conceived singleton whose parents were nonconsanguineous Koreans. Her mother was a 34-year-old multigravida with a history of two artificial abortions and one spontaneous abortion. The findings of antenatal ultrasound at a gestational age of 27 weeks and 2 days were suggestive of IUGR and fetal anomalies, including cardiomegaly, pyelectasis, and focal bowel dilatation. Maternal placental biopsy did not reveal signs of inflammation, infarction, or structural abnormalities (Fig. 1). The parents reported no family history of genetic syndromes. The infant's birth weight, length, and head circumference were $720 \mathrm{~g}(-1.12$ standard deviation [SD]), $30 \mathrm{~cm}(-2.19$ SD), and $23.5 \mathrm{~cm}$ (-0.86 SD), respectively (Fig. 2) [5]. Physical examination showed dysmorphic facial features, including a broad forehead, downward slanted palpebral fissures, small pointed chin, and large low-set ears. The Apgar scores were 1 at 1 minutes and 2 at 5 minutes. She was immediately intubated and received a surfactant after birth. Thereafter, she was admitted to the neonatal intensive care unit (NICU). She was maintained on mechanical ventilator support for 35 days, followed by oxygen supplementation via a nasal cannula and hood for an additional 43 days. She was treated for bronchopulmonary dysplasia and secondary pulmonary hypertension. She also had other medical

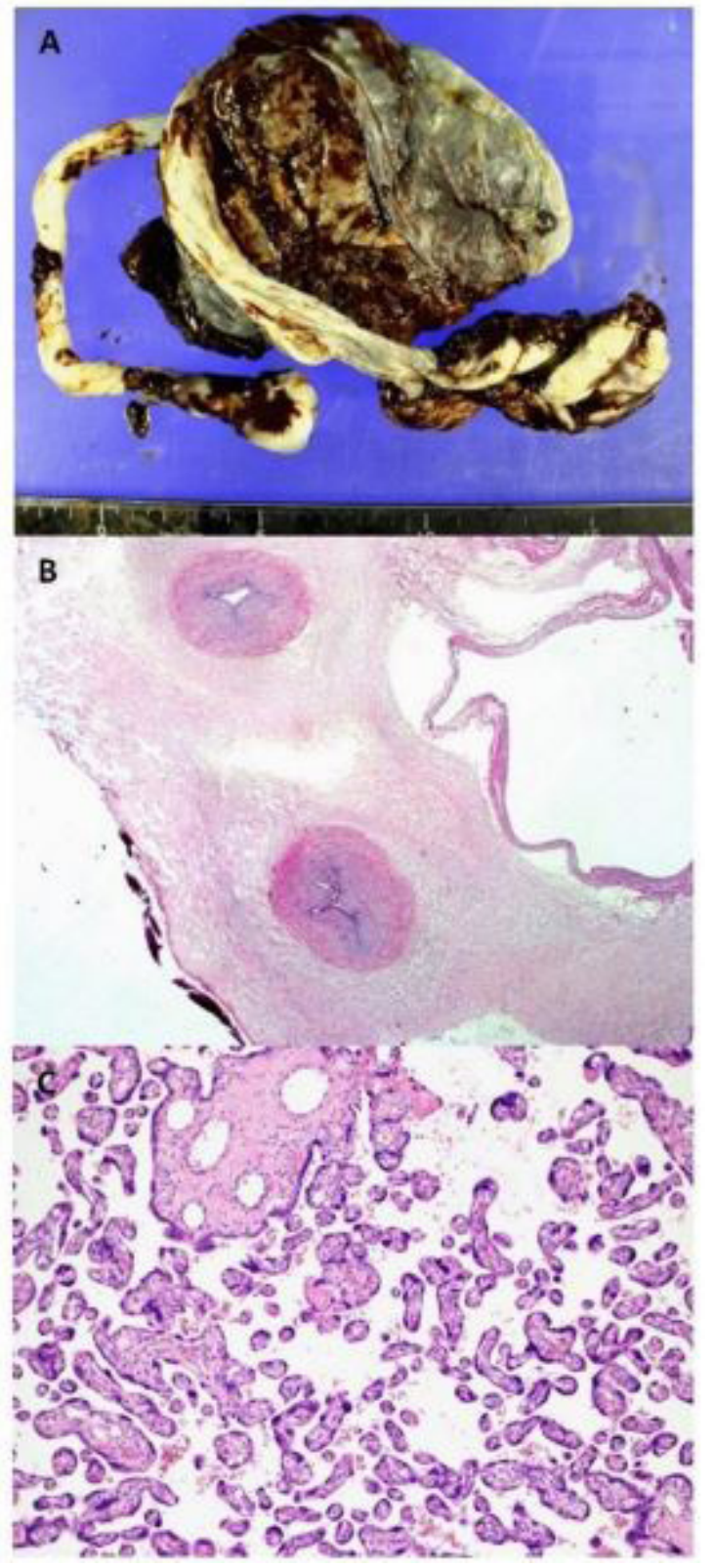

Fig. 1. Maternal placental biopsy of the patient. (A) Gross morphology showing no gross abnormality of the maternal placenta. (B) Microscopic view of the umbilical cord showing normal vascular structure without signs of inflammation (hematoxylin-eosin [H\&E] staining; magnification, $\times 12.5)$. (C) Microscopic view of the placental parenchyma showing no vasculopathy or infarction (H\&E staining; magnification, $\times 100)$.

issues, including patent ductus arteriosus, atrial septal defect, retinopathy of prematurity, congenital hypothyroidism, sepsis, meningitis, and unilateral inguinal hernia.

Owing to feeding difficulties because of poor appetite and oromotor issues, the infant was fed via a nasogastric tube and was administered oromotor stimulation therapy daily. Owing to poor weight gain, we coordinated with the nutrition sup- 
port team on a weekly basis to optimize her growth. She was fed a high-calorie fortified formula, and her daily caloric intake was closely monitored. However, at 40+0 weeks of postmenstrual age, she continued to show postnatal growth failure; her weight, length, and head circumference were 1,920 g (-3.74 SD), $49 \mathrm{~cm}$ (-2.69 SD), and $31 \mathrm{~cm}$ (-0.63 SD), respectively (Fig. 2) [5].

We assessed the possibility of a genetic cause of IUGR, postnatal growth failure, feeding difficulties, and facial dysmorphic features. As these four features correspond to the characteristic phenotype of SRS, we conducted the following first-line molecular tests for SRS: MS-MLPA for chromosome 11 and MS-PCR restriction fragment length polymorphism (RFLP) analysis for chromosome 7. MS-MLPA was performed at the LITI-differentially methylated region (DMR) and H19-DMR of chromosome 11. The methylation patterns for the 7q.21.3 region (SGCE gene) were analyzed using PCR products of bisulfite-modified SGCE. MS-MLPA showed normal methylation patterns of $11 \mathrm{p} 15$; however, MS-PCR RFLP amplified only the maternal allele, sug-

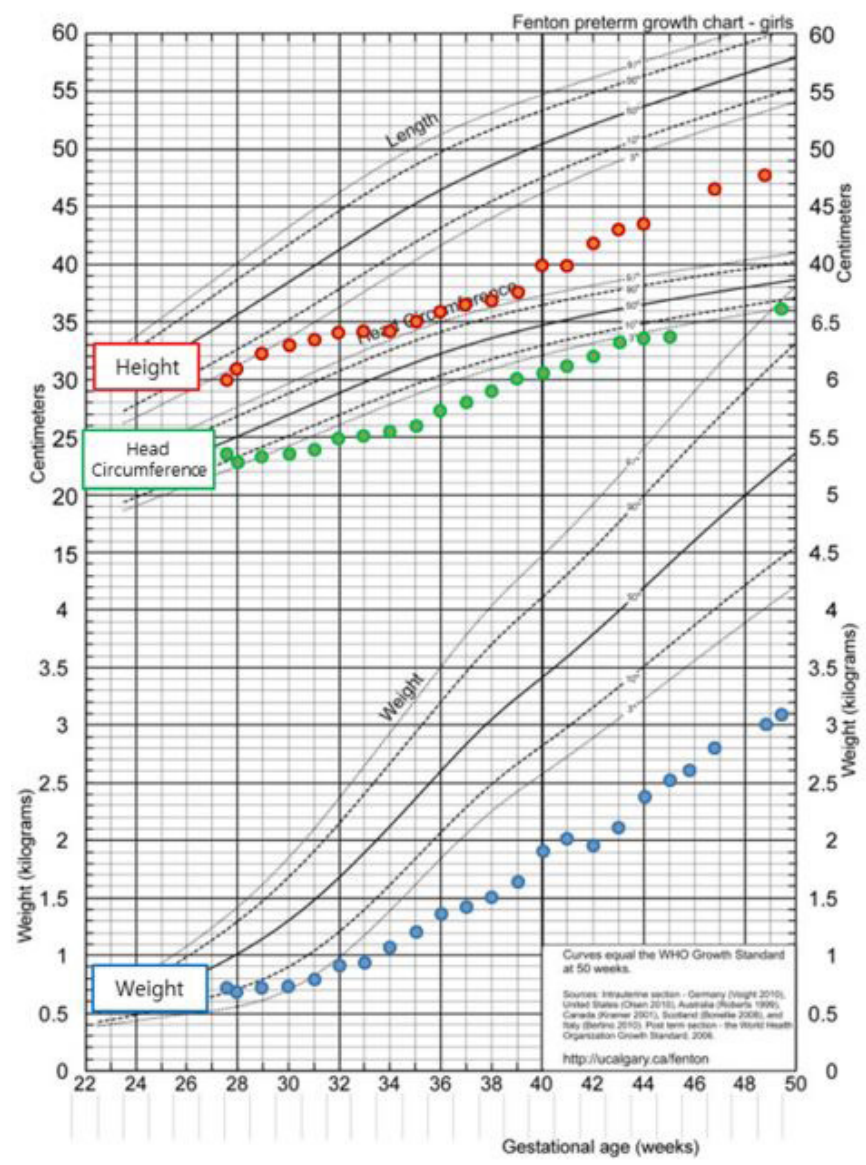

Fig. 2. Growth curve of the patient. The growth curve of the patient shows intrauterine growth retardation and postnatal growth failure without catch-up [5]. gesting UPD(7)mat (Fig. 3A, B). Chromosomal microarray (CMA) analysis was also performed to confirm the molecular diagnosis of SRS and identify other possible genetic disorders and chromosomal aberrations. CytoScan Dx assay (Thermo Fisher Scientific Inc., Waltham, MA, USA) was used, which combines array-based comparative genomic hybridization and single nucleotide polymorphism (SNP) genotyping. CMA analysis did not reveal the copy number variation in the 11 p15 and $7 q 21$ regions or loss of heterozygosity in the $7 q 21$ region. A duplication of Xp22.2p22.13 was detected on CMA analysis (Fig. 3C). The
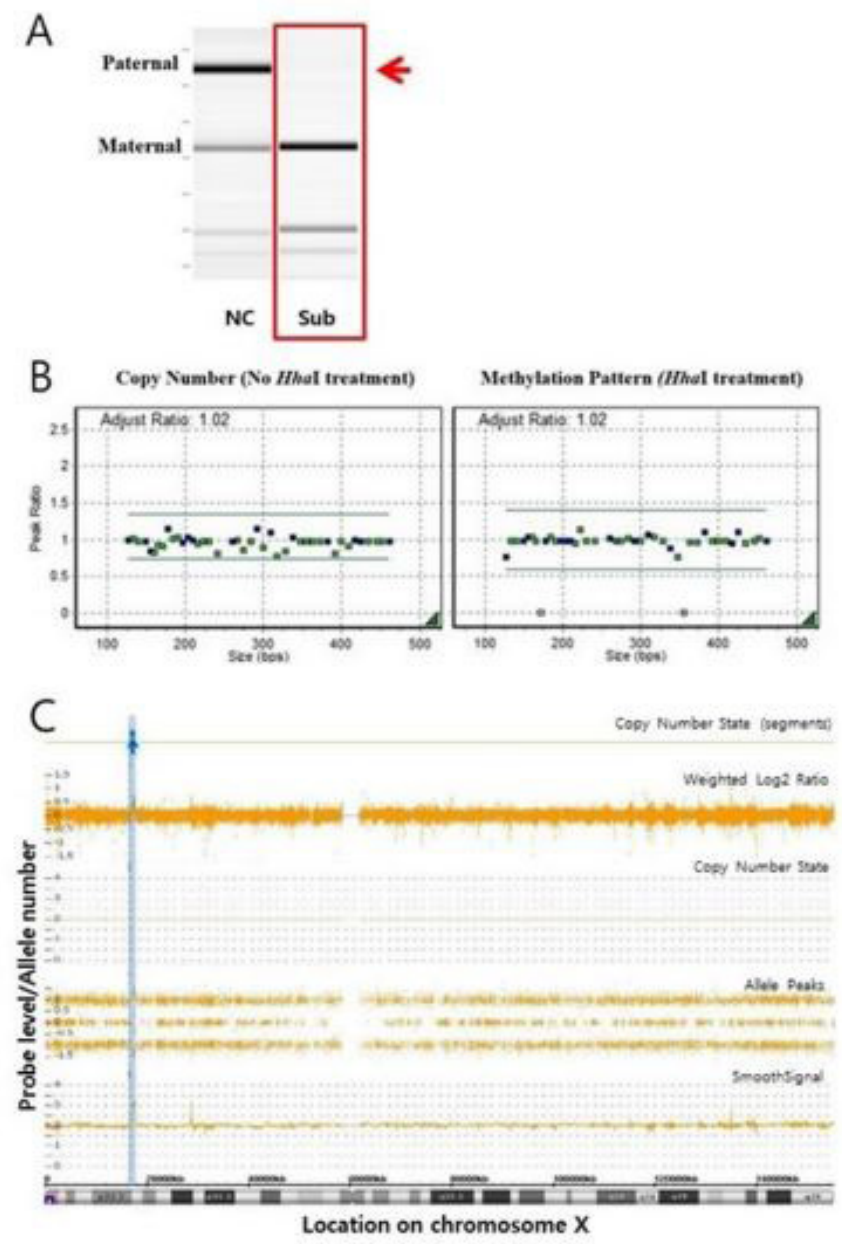

Fig. 3. Genetic testing of the patient. (A) MS-PCR RFLP analysis of the $7 q .21 .3$ region $(S G C E$ ) revealed UPD(7)mat in the patient. The arrow indicates the missing paternal section. (B) MS-MLPA analysis of $11 p 15$ showed a normal methylation pattern and the absence of copy number variation. (C) Copy number gain at Xp22.2p22.13 (16,985,921$17,731,098 \mathrm{~kb}$ ) was detected on chromosomal microarray analysis (the duplicated region is indicated by the blue arrow with light blue outline). The $X$-axis presents the probe index on chromosome $X$, and the Y-axis presents the signal $\log 2$ ratio of the probe. MS-PCR RFLP, methylation-specific polymerase chain reaction restriction fragment length polymorphism; UPD(7)mat, maternal uniparental disomy of chromosome 7; MS-MLPA, methylation-specific multiplex ligation probe-dependent analysis; Sub, subject; NC, normal control. 
duplication was classified as a variant of uncertain significance (VOUS).

After the diagnosis, we conducted a surveillance medical examination and provided education and genetic counseling to the patients. We also established management plans for the short stature and low body mass index (BMI) of the infant; the plans included provision of adequate nutritional support and initiation of growth hormone therapy at the optimal time. We educated the mother regarding the increased risk for hypoglycemia, precocious puberty, developmental delay, and learning difficulties noted in children with SRS with UPD(7)mat. The patient was discharged from the NICU at 165 days after birth at the postmenstrual age of $46+0$ weeks. She required nasogastric tube feeding at times, but her general medical condition was well otherwise. At the age of 7 months, her height and weight were $55.7 \mathrm{~cm}$ (below -3 SD) and $4.5 \mathrm{~kg}$ (below -3 SD), respectively. Her serum insulin-like growth factor 1 (IGF-1) and IGFbinding protein 3 levels at the age of 7 months were $112.7 \mathrm{ng} /$ $\mathrm{mL}$ (reference range: $5.1-186.5 \mathrm{ng} / \mathrm{mL}$ ) and 2,560 ng/mL (reference range: $950-2,120 \mathrm{ng} / \mathrm{mL}$ ), respectively [6]. We plan to assess her growth and neurocognitive development in the upcoming visits to the hospital to ensure that appropriate interventions are provided.

\section{Discussion}

IUGR is defined as the rate of fetal growth being less than the normal growth rate based on the growth potential of a specific infant. The diagnosis is based on at least two ultrasonography measurements performed at least 2 weeks apart, with the fetal weight below the 10th percentile for the gestational age [1]. The etiology of IUGR is extremely heterogeneous and may involve maternal, placental, and fetal factors [7]. Genetic anomalies in the fetus account for $5-20 \%$ of the IUGR cases. These anomalies include chromosomal aberrations, imprinting disorders, and other genetic syndromes such as SRS, Bloom syndrome, Cornelia de Lange syndrome, Mulibrey nanism syndrome, and RubensteinTaybi syndrome $[8,9]$. Although most genetic syndromes with chromosomal aberrations can be detected with CMA analysis, some imprinting disorders, such as SRS, may yield normal results on CMA testing, and specific molecular testing is required for the diagnosis of these disorders.

SRS is characterized by IUGR, poor postnatal growth failure, relative macrocephaly, a triangular face, body asymmetry, and feeding difficulties. The syndrome was first described by Silver et al. [10] and Russell [11], who independently described a group of children with low birth weight, postnatal short stature, characteristic facial features, and body asymmetry. In 2015, Azzi et al. [2] proposed the NH-CSS for SRS. The clinical diagnosis of SRS can be established in an individual who meets at least four of the six NH-CSS clinical criteria, including prominent forehead/ frontal bossing and relative macrocephaly at birth and two additional findings, and in whom other disorders have been ruled out. The other criteria are small for gestational age/IUGR, postnatal growth failure, body asymmetry, and feeding difficulties/ low BMI. The supporting clinical findings of SRS include delayed closure of the anterior fontanelle (43\%), triangular face (94\%), micrognathia (62\%), dental crowding (37\%), down-turned corner of the mouth (48\%), high-pitched voice (45\%), decreased muscle mass (56\%), skeletal abnormalities, developmental delay, and genitourinary anomalies [1]. Patients with SRS also develop endocrine issues, including fasting hypoglycemia, premature adrenarche, early puberty, and insulin resistance. The possible skeletal abnormalities of SRS include asymmetry with respect to the limb length, fifth-finger clinodactyly, and scoliosis. In our case, the premature infant presented with IUGR, postnatal growth failure, feeding difficulties, and a triangular face with a broad forehead and micrognathia. Asymmetry in the leg length was clinically noted (discrepancy of $0.5 \mathrm{~cm}$ ) but not confirmed on radiography. However, the infant showed no signs of scoliosis, clinodactyly, or genitourinary anomalies.

Considering the subjective nature of the clinical diagnosis, molecular genetic identification of SRS is particularly important [12]. Molecular testing also defines the genetic subtype of SRS, which can guide appropriate management of patients with different genotypes. The major molecular changes are LOM of 11 p 15 and UPD(7)mat. Other rare etiologies include copy number variations on chromosome 7 or 11, or an intragenic pathogenic variant in CDKN1C, IGF2, PLAG2, or HMGA2 [13]. Methylation-specific analysis for both chromosomes 7 and 11 should be considered in patients whose phenotype is consistent with SRS. MS-PCR for UPD(7)mat and MS-MLPA for 11 p15 LOM have been recommended as first-line testing $[3,4]$. The methylation defects of H19-DMR and LITI-DMR (previously known as ICR 1 and 2) can be detected with MS-MLPA of 11 p15 $[1,3]$. Hypomethylation of the H19-DMR in $11 \mathrm{p} 15$ results in reduced paternal IGF2 expression and increased maternal H19 expression, leading to growth restriction [1].

UPD is the presence of a chromosome pair derived only from one parent in a disomic cell line [14]. The phenotype of SRS patients with UPD(7)mat is believed to result from altered expressions of the imprinted growth and developmental regulatory 
genes. GRB10 and MEST have been suggested as the candidate genes on chromosome 7 accounting for the development of SRS [4]. Patients with UPD(7)mat generally show a milder phenotype than patients with LOM of 11p15. Micrognathia, low set ears, learning difficulties, and speech disorders have been more frequently reported in patients with UPD(7)mat [15] However, pathognomonic features distinguishing between the UPD(7) mat genotype and other genotypes have not been reported $[3,16,17]$. In cases of UPD $(7)$ mat identified with MS-PCR, the diagnosis can be confirmed by performing microsatellite analysis with the DNA from at least one parent. We identified UPD(7) mat in the patient; however, we could not perform microsatellite analysis, as the parents refused parental testing. Although abnormal methylation patterns may not be detected with CMA analysis in some cases, CMA analysis can still provide useful information for the classification of UPD [18]. Maternal UPD can be detected using CMA with SNP array analyses only in cases of isodisomy [19]. Additionally, in one-third of the cases, UPD develops in association with or as a result of a chromosomal rearrangement [14]. In our case, CMA with SNP array analysis did not reveal copy number variation or loss of heterozygosity in the $7 q 21$ region, which implies that UPD(7)mat developed in the infant because of heterodisomy. In this case, a 745-kb duplication at Xp22.2p22.13 (16,985,921-17,731,098) was detected on CMA analysis. The duplicated region involves some of the exons in the NHS gene. Mutation in the NHS gene can lead to the development of Nance-Horan syndrome through loss of gene function. Nance-Horan syndrome is a rare $\mathrm{X}$-linked recessive disease characterized by severe congenital dense nuclear cataracts and developmental delay in hemizygous male patients. Carrier females can display milder symptoms [20]. The infant in our case was a girl with duplication, whose lenses appeared normal on ophthalmologic examination. Thus, the variation was suspected to be VOUS, which would have been confirmed if parental testing was performed.

In 2017, Wakeling et al. [1] published the first international consensus statement on the diagnosis and management of SRS, which emphasized adequate nutritional status with the avoidance of rapid and excessive weight gain. Early initiation of growth hormone therapy benefits patients by improving the body composition, motor development, and appetite, thus, optimizing linear growth and reducing the risk of hypoglycemia. Patients should be monitored for signs of premature adrenarche, early and rapid central puberty, and insulin resistance. Gonadotropin-releasing hormone analogues can delay the progression of central puberty and preserve the potential to achieve the op- timal adult height in the future. For our patient, we established a specific management plan and provided educated the parent regarding nutritional support, recurrence risk, and possible medical issues, including neurocognitive problems, endocrine problems, surgical/anesthesia issues, orthopedic problems, and maxillofacial abnormalities. Owing to early diagnosis, we were able to provide appropriate management and parental education for each subtype of SRS. The parents were informed that the recurrence risk was low because of the de novo development of UPD(7)mat [1]. Three weeks after discharge, the infant was readmitted to the hospital for surgical repair of an inguinal hernia. Before being sent to the operating room, she was intubated by the NICU team owing to difficulties associated with visualizing the airway and intubating SRS patients with small mandibles.

Identification of the underlying molecular subtype can influence the management strategies with respect to the specific risk factors associated with each subtype; however, this can be challenging. We report a case of SRS with suspected uniparental heterodisomy of chromosome 7 in an ELBW infant. SRS is a rare genetic cause of IUGR, and ELBW infants have several risk factors that may contribute towards feeding difficulties and postnatal growth failure; however, when relative macrocephaly, prominent forehead, or body asymmetry is noted in patients with IUGR, clinicians should suspect SRS. Once SRS is suspected, appropriate methylation-specific genetic testing should be conducted, the abnormal methylation patterns may not be detected on performing CMA analysis or other molecular genetic tests. With this report we aim to improve the understanding of the complex molecular etiology of SRS and aid clinicians in establishing accurate diagnoses early on, thereby, promoting better outcomes in patients with SRS.

\section{References}

1. Wakeling EL, Brioude F, Lokulo-Sodipe O, O'Connell SM, Salem J, Bliek J, et al. Diagnosis and management of Silver-Russell syndrome: first international consensus statement. Nat Rev Endocrinol 2017;13:105-24.

2. Azzi S, Salem J, Thibaud N, Chantot-Bastaraud S, Lieber E, Netchine I, et al. A prospective study validating a clinical scoring system and demonstrating phenotypical-genotypical correlations in SilverRussell syndrome. J Med Genet 2015;52:446-53.

3. Eggermann $T$, Begemann $M$, Binder $G$, Spengler S. Silver-Russell syndrome: genetic basis and molecular genetic testing. Orphanet J Rare Dis 2010;5:19.

4. Kim Y, Kim SS, Kim G, Park S, Park IS, Yoo HW. Detection of maternal uniparental disomy at the two imprinted genes on chromosome 7 , 
GRB10 and PEG1/MEST, in a Silver-Russell syndrome patient using methylation-specific PCR assays. Clin Genet 2005;67:267-9.

5. Fenton TR, Kim JH. A systematic review and meta-analysis to revise the Fenton growth chart for preterm infants. BMC Pediatr 2013;13:59.

6. Korean Society of Pediatric Endocrinology. Pediatric Endocrinology. 3rd ed. Seoul: Koonja; 2014.

7. Suhag A, Berghella V. Intrauterine growth restriction (IUGR): etiology and diagnosis. Curr Obstet Gynecol Rep 2013;2:102-11.

8. Sharma D, Shastri S, Farahbakhsh N, Sharma P. Intrauterine growth restriction - part 1. J Matern Fetal Neonatal Med 2016;29:3977-87.

9. Stalman SE, Solanky N, Ishida M, Alemán-Charlet C, Abu-Amero S, Alders $M$, et al. Genetic analyses in small-for-gestational-age newborns. J Clin Endocrinol Metab 2018;103:917-25.

10. Silver HK, Gruskay FL. Syndrome of congenital hemihypertrophy and elevated urinary gonadotropins; occurrence in a seven-year-old boy. AMA J Dis Child 1957;93:559-62.

11. Russell A. A syndrome of intra-uterine dwarfism recognizable at birth with cranio-facial dysostosis, disproportionately short arms, and other anomalies (5 examples). Proc R Soc Med 1954;47:1040-4.

12. Eggermann T. Russell-Silver syndrome. Am J Med Genet C Semin Med Genet 2010;154C:355-64.

13. Neuheuser $L$, Meyer $R$, Begemann $M$, Elbracht M, Eggermann T. Next generation sequencing and imprinting disorders: current applications and future perspectives: lessons from Silver-Russell syndrome. Mol

\section{Cell Probes 2019:44:1-7.}

14. Liehr T. Cytogenetic contribution to uniparental disomy (UPD). Mol Cytogenet 2010;3:8.

15. Kotzot D. Maternal uniparental disomy 7 and Silver-Russell syndrome - clinical update and comparison with other subgroups. Eur J Med Genet 2008;51:444-51.

16. Wakeling EL, Amero SA, Alders M, Bliek J, Forsythe E, Kumar $S$, et al. Epigenotype-phenotype correlations in Silver-Russell syndrome. J Med Genet 2010;47:760-8.

17. Lee BH, Kim GH, Oh TJ, Kim JH, Lee JJ, Choi SH, et al. Quantitative analysis of methylation status at $11 p 15$ and $7 q 21$ for the genetic diagnosis of Beckwith-Wiedemann syndrome and Silver-Russell syndrome. J Hum Genet 2013;58:604-10.

18. Tucker T, Schlade-Bartusiak K, Eydoux P, Nelson TN, Brown L. Uniparental disomy: can SNP array data be used for diagnosis? Genet Med 2013;14:753-6.

19. Chantot-Bastaraud $S$, Stratmann $S$, Brioude F, Begemann M, Elbracht $M$, Graul-Neumann $L$, et al. Formation of upd(7)mat by trisomic rescue: SNP array typing provides new insights in chromosomal nondisjunction. Mol Cytogenet 2017;10:28.

20. Brooks SP, Ebenezer ND, Poopalasundaram S, Lehmann OJ, Moore AT, Hardcastle AJ. Identification of the gene for Nance-Horan syndrome (NHS). J Med Genet 2004;41:768-71. 